\title{
Das habsburgische Kaisertum und die Fürstpropstei Ellwangen in der Frühen Neuzeit
}

\author{
Von Franz Brendle
}

\section{Die Fürstpropstei Ellwangen im frühneuzeitlichen Reich ${ }^{*}$}

\begin{abstract}
„Nachdem Gott der Allmächtige dieses hochfürstliche Stift innerhalb von tausend von dessen Errichtung bis anhero verflossenen Jahren nicht allein bis auf diese Stund gütigst erhalten, sondern auch von vielfältigen Unfällen solche Zeit hindurch - ohne Zweifel auf die mächtige Fürbitt unserer heiligen Stifts- und Schutzpatrone - gnädigst bewahrt und zu noch größerem Wachstum befördert, als hat man sich verpflichtet zu sein erachtet, dem Allerhöchsten vordersamst und sodann auch diesen unseren Schutzheiligen durch eine 8 tägige Feierlichkeit den schuldigsten Dank öffentlich [...] abzustatten. " ${ }^{\text {"S }}$ So lautete die Einladung, die das Ellwanger Stiftskapitel an die Ellwanger Bevölkerung aussprach, um die Jahrtausendfeier der Ellwanger Kirche festlich zu begehen². Über eine Woche, nämlich vom 18. bis zum 26. August 1764 sollte die Feierlichkeit dauern, um dem Allmächtigen Dank dafür zu sagen, dass er das Stift über die Jahrhunderte hinweg seit seiner Gründung geführt und bewahrt hatte. Die reiche Geschichte Ellwangens wurde dargestellt in einer zum Anlass des Jubiläums errichteten Triumphpforte, die sich zu einem Hauptanziehungspunkt der Feier entwickelte. In diesen Worten klingt schon der tiefere Sinn einer solchen Feierlichkeit an: Dank zu sagen für die Führung und Bewahrung über ein Jahrtausend hinweg, für die organische Tradition, für eine reiche Geschichte. Diese Geschichte wurde dargestellt in einer zum Anlass des Jubiläums errichteten Triumphpforte, die zum Hauptanziehungspunkt der Feier wurde.
\end{abstract}

* Der Beitrag basiert auf dem öffentlichen Abendvortrag, der im Rahmen der 63. Jahrestagung der Kommission für geschichtliche Landeskunde in Baden-Württemberg am 30. Juni 2016 in Ellwangen gehalten wurde.

1 Zitiert nach Alois SEILER, Die Jahrtausendfeier des Stifts Ellwangen im Jahre 1764, in: Ellwanger Jahrbuch $20(1962 / 64)$ S. 8.

${ }^{2}$ Vgl. dazu ebd., S.7-19. 
Das Innere der Triumphpforte veranschaulichte die wechselvolle Geschichte des Ellwanger Stifts ${ }^{3}$. Die östliche Wand war den unglücklichen Ereignissen gewidmet, den zahlreichen Bränden des Klosters, der Heimsuchung durch die Pest, dem Bauernkrieg und der schwedischen Besetzung des Stifts während des Dreißigjährigen Kriegs. Auf der westlichen Wand dagegen wurden die glücklichen Ereignisse der ellwangischen Geschichte abgebildet. In diese Bildfolge wurden nicht nur die Grundsteinlegungen der Ellwanger Kirchen und die Niederlassungen der kirchlichen Orden, sondern auch die Privilegien der mittelalterlichen Kaiser für das Kloster Ellwangen aufgenommen - Zeichen für das Wohlwollen und den Schutz, den das Reichsoberhaupt über Jahrhunderte hinweg für Kloster und Stift Ellwangen geleistet hatte. In der weltlichen Sphäre galt der Kaiser als der Garant für den glücklichen Verlauf der Ellwanger Geschichte.

Anhand dreier Beispiele soll im Folgenden die enge Verbindung zwischen dem habsburgischen Kaisertum und der Fürstpropstei Ellwangen aufgezeigt werden, wobei nicht immer das Kaisertum als Schutzherr des Stifts auftrat:

1. In den Auseinandersetzungen der Reformationszeit,

2. den kaiserlichen Kommissionen und dem kaiserlichen Besetzungsrecht im Stiftskapitel und

3. dem kaiserlichen Einfluss auf die Propstwahlen.

Einige wenige Bemerkungen zur Entwicklung von Kloster und Stift Ellwangen sollen am Anfang stehen ${ }^{4}$. Bereits Ludwig der Fromme verlieh der Abtei 814 ein Immunitätsprivileg und gestattete ihr die freie Abtswahl. Ein päpstlicher Schutzbrief von 979 wurde immer wieder als Exemtionsprivileg gegen die bischöflichen Ansprüche interpretiert. Im 13. Jahrhundert stieg der Ellwanger Abt in den Reichsfürstenstand auf, seit dem 14. Jahrhundert werden seine Unabhängigkeit vom Diözesanbischof in Augsburg und seine Papstunmittelbarkeit dadurch unterstrichen, dass er sich von einem Bischof seiner Wahl weihen lassen konnte ${ }^{5}$. Der Auskauf der alten Vogteirechte von den Grafen von Oettingen legte im 14. Jahrhundert die Grundlage für eine eigene Landesherrschaft. Gestützt wurde diese Entwicklung vom regionalen Niederadel, der seine eigene Existenz mit der des Klosters verknüpfte. Grundlage der Territorialherrschaft waren jedoch die arrondierten

3 Ebd., S. 17 ff.

${ }^{4}$ Zur Geschichte von Kloster und Stift Ellwangen vgl. Viktor Burr (Hg.), Ellwangen 764-1964. Beiträge und Untersuchungen zur Zwölfhundertjahrfeier, 2 Bde., Ellwangen 1964; Volker Press, Ellwangen, Fürststift im Reich des späten Mittelalters und der frühen Neuzeit, in: Ellwanger Jahrbuch 30 (1983/84) S.7-30.

${ }_{5}^{5}$ Eugen Heinrich Fischer, Ellwangen, Augsburg, Rom. Die Exemtion des Ellwanger Stifts und seine Exemtionspolitik unter Fürstpropst Franz Georg von Schönborn in den Jahren 1732 bis 1749, in: BurR, Ellwangen 764-1964, Bd.1 (wie Anm. 4) S.379-423. 
Grundherrschaften im Nahbereich der Herrschaft mit Kloster und Stadt ${ }^{6}$. Gleichzeitig entstanden neue schutzherrschaftliche Bindungen. 1370 übertrug der Kaiser Schutz und Schirm Ellwangens an Württemberg, das damit ein wichtiges Einfallstor öffnen konnte. In Übereinstimmung mit dem Wiener Konkordat von 1448 wurde dem Kapitel unter der Führung des Dekans bei der Umwandlung die freie kanonische Propstwahl bestätigt.

Unter württembergischer Regie erfolgte im 15. Jahrhundert der Versuch einer inneren Klosterreform, die jedoch an den Standes- und Versorgungsinteressen des Adels scheiterte. Der Niedergang des klösterlichen Lebens wurde schließlich sanktioniert, indem Abt und Konvent beim Papst die förmliche Umwandlung in ein adeliges, weltliches Stift ersuchten. Am 14. Januar 1460 stimmte Papst Pius II. diesem Vorhaben zu, die kaiserliche Bestätigung erfolgte im Dezember desselben Jahres ${ }^{7}$.

Mit der Aufhebung der Ordensregel wurde ein weltliches Kollegiatstift mit zwölf Kanonikaten nach dem Vorbild der Apostelzahl eingerichtet. Der Fürstpropst stand als Dreizehnter an der Spitze des Chorherrenstifts. Er besaß als weltlicher Herr die Regalien und bekleidete den Rang eines Fürsten mit Sitz und Stimme auf dem Reichstag. Im Reichstag saß der Fürstpropst als 29. Stand auf der geistlichen Fürstenbank hinter allen Bischöfen, den Fürstäbten von Fulda und Kempten, unmittelbar vor dem Johanniterordensmeister und dem Fürstpropst von Berchtesgaden. Auf den Konventen des Schwäbischen Kreises alternierte der Vorrang mit Kempten. Der Ellwanger Fürstpropst verfügte über quasi-bischöfliche Rechte. Seine Kleidung entsprach dem Bischofstalar, als Insignien standen ihm Mitra, Ring und Stab zu, ebenso eine Kathedra in der Stiftskirche. Wie der Bischof hatte auch der Fürstpropst bestimmte Voraussetzungen zu erfüllen: ein Mindestalter von 30 Jahren, die Priesterweihe und den theologischen oder juristischen Doktorgrad. Allerdings ließen sich besonders für hochadelige Bewerber sehr leicht Dispense erlangen. Die Attraktivität des Stifts für die hochadeligen Dynastien rührte auch von der relativen Prosperität her, die den Einkünften eines kleineren Bistums entsprach.

\section{Kaiser Karl V. und der Deutschmeisterkrieg}

Es blieb eine Belastung, dass das Fürststift zwar wohlhabend war, aber doch auch sehr peripher gelegen, vor allem gegen das ausgreifende Württemberg. Dies wurde gerade im Reformationszeitalter zu einem Problem, als das Stift seine

${ }^{6}$ Zur Verfassung des Stifts: Hans Pfeifer, Verfassungs- und Verwaltungsgeschichte der Fürstpropstei Ellwangen, Stuttgart 1959.

7 Joseph Zeller, Die Umwandlung des Benediktinerklosters Ellwangen in ein weltliches Chorherrenstift (1460) und die kirchliche Verfassung des Stifts, Stuttgart 1910; Hans PfeIFER, Vom Benediktinerstift Ellwangen zum weltlichen Chorherrnstift (1460), in: Ellwanger Jahrbuch 31 (1985/1986) S.53-66. 
Selbständigkeit gegen den Kaiser und Württemberg verteidigen musste ${ }^{8}$. Ausgangspunkt der Auseinandersetzungen war der Übergang des Hochmeisters des Deutschen Ordens, Albrecht von Brandenburg, 1525 zur Reformation. Das Deutschordensland in Ostpreußen wurde in ein weltliches Herzogtum umgewandelt, als erstes geistliches Territorium im Reich, sein Besitz ging damit dem Deutschen Orden verloren.

Zwar konnte der Deutschmeister, der seit der Zerstörung der Burg Horneck im Bauernkrieg in Mergentheim residierte, als Administrator des Hochmeistertums die Aufgaben des Ordensoberen weiterführen, doch war Wolfgang Schutzbar, genannt Milchling, der 1542 in das Amt gewählt worden, daran gelegen, eine seiner Stellung angemessene Ausstattung zu erhalten ${ }^{9}$. Der mit dieser Aufgabe betraute Kanzler des Deutschmeisters, Gregor Spieß, stellte daraufhin eine Denkschrift an Schutzbar zusammen, in der er folgenden Vorschlag machte: „Mein Herr Statthalter und ich haben uns miteinander besprochen und wir haben unsere Überlegungen auf die Propstei Ellwangen gerichtet, weil wir im ganzen Reich kein Ding wüßten, das für den Orden feiner und gelegener wäre als ebendiese Propstei. “10

Und er fährt fort: „Wenn Euer fürstliche Gnaden diese Propstei für den Orden erlangen könnten, wenn sie ihm inkorporiert würde, und wenn Euer fürstlich Gnaden und dero Nachfolger dann ihren Sitz und Residenz dort nehmen würden, dann stünde solcher Stuhl mitten in der reichsten Ordenslandschaft [...] Es ist dort eine feine Landschaft mit aller fürstlichen Herrlichkeit und Obrigkeit. Das Land ist voll von Wildbret, Fisch, Fleisch und aller anderen notwendigen Dinge, ausgenommen Wein, es hat etliche gute und feine Schlösser und Ämter [...] Und es hat viele gute und ehrliche Leute vom Adel zu Lehensleuten. Der Propst ist zugleich ein Fürst des Reiches und besitzt seine Regalien, und in geistlichen Sachen ist er, wie Euer Gnaden, niemandem sonst als dem Papst unterworfen [...] Soweit der mergentheimische Kanzler. Er fügt noch an: Der Mangel an Wein könnte leicht und reichlich aus den Ordensämtern an Tauber und Neckar ersetzt werden.

Der Deutschmeister Wolfgang Schutzbar war von diesem Vorschlag überzeugt und versuchte ihn zunächst mit diplomatischen Mitteln umzusetzen. In Ellwangen regierte seit 1521 Pfalzgraf Heinrich als Fürstpropst, der gegen den Widerstand des Stiftskapitels vom Kaiser und der Kurpfalz dem Stift regelrecht aufgezwungen worden war. Er zählte zu einem der erfolgreichsten Pfründenjäger in der Reichskirche, konnte er doch nicht nur in Ellwangen, sondern auch in Worms, Utrecht und zuletzt 1540 in Freising reüssieren. Der Pfalzgraf stand dem Plan des Deutschmeis-

${ }^{8}$ Vgl. dazu Dieter Stievermann, Das geistliche Fürstentum Ellwangen im 15. und 16. Jahrhundert: Politische Selbstbehauptung im Schatten Württembergs, in: Ellwanger Jahrbuch 32 (1987/1988) S. 35-47.

9 Vgl. dazu Alois SeILer, Württemberg in der Auseinandersetzung mit dem Deutschen Orden. Der „Deutschmeisterkrieg“ von 1552, in: Beiträge zur Kulturgeschichte von Altshausen und Umgebung 13 (1990) S.35-43.

10 Dieses und die folgenden Zitate nach SEILER, Württemberg (wie Anm. 9). 
ters zumindest anfänglich positiv gegenüber, zumal bei seinen zahlreichen Ämtern die Unterstützung eines Koadjutors durchaus nützlich sein konnte. Die Zustimmung Kaiser Karls V. hatte in erster Linie religiöse Gründe. Bei einer Regentschaft des streng altgläubigen Deutschmeisters und der Vereinigung Mergentheims und Ellwangens konnte das Stift an der Jagst sehr viel sicherer beim alten Glauben gehalten werden. Von württembergischer Seite waren dagegen eher Gefahren zu erwarten, da Herzog Ulrich von Württemberg als Schirmherr des Stifts seit 1534 in seinem Herzogtum konsequent die Reformation durchsetzte ${ }^{11}$.

In Gent entwarf die kaiserliche Kanzlei ein Memorandum, um Propst und Kapitel mit der Absicht vertraut zu machen, zur Erhaltung des Gottesdienstes das Stift rechtzeitig mit einer „tapferen, ansehnlichen Person, von Namen, Jugend und Geschicklichkeit“ zu versehen. Von Jugend konnte bei Schutzbar zwar nur noch bedingt die Rede sein - er war zu diesem Zeitpunkt immerhin schon 65 Jahre alt -, doch Fürstpropst Heinrich erwies sich sehr zugänglich und war sogar bereit, die Propstei dem Deutschmeister ganz zu resignieren. Vom 28. April 1545 datiert eine notarielle Urkunde, welche die förmliche Resignation zugunsten von Wolfgang Schutzbar vollzog. Doch verweigerte das Kapitel seine Zustimmung, trotz intensiver kaiserlicher Werbungen. Als Begründung gaben die Stiftsherren an, das kaiserliche Vorgehen widerspreche den hergebrachten und gültigen Statuten des Stifts, das heißt, es verletze das freie Wahlrecht des Kapitels. Dazu kam die Befürchtung - und so unbegründet war sie nicht - die Propstei könnte dem Deutschen Orden inkorporiert werden. Dadurch würde dem niederen Adel seine Versorgung entzogen. Denn was der Deutsche Orden einmal habe, das gebe er nicht mehr her.

In ihrer bedrängten Situation hatten sich die Ellwanger Stiftsherren auch an Herzog Ulrich von Württemberg als Schutz- und Schirmherrn der Propstei gewandt ${ }^{12}$. Auch von Landgraf Philipp ${ }^{13}$, der seinerseits mit Pfalzgraf Heinrich in Verbindung

$11 \mathrm{Zu}$ Herzog Ulrich vgl. Volker Press, Herzog Ulrich (1498-1550), in: Robert UhLAND (Hg.), 900 Jahre Haus Württemberg. Leben und Leistung für Land und Volk, Stuttgart ${ }^{3} 1985$, S.110-135; Franz Brendle, Dynastie, Reich und Reformation. Die württembergischen Herzöge Ulrich und Christoph, die Habsburger und Frankreich, Stuttgart 1998; Ders., Herzog Ulrich - ein verkannter Reformationsfürst, in: Siegfried Hermle (Hg.), Reformationsgeschichte Württembergs in Porträts, Holzgerlingen 1999, S. 199-225; Ders., Ulrich, Hzg. v. Württemberg, in: LThK 10 (2000), Sp. 359-360; Ders., Ulrich von Württemberg (14871550), in: Susan Richter/Armin Kohnle (Hg.), Herrschaft und Glaubenswechsel. Die Fürstenreformation im Reich und in Europa in 28 Biographien, Heidelberg 2016, S. 146-163.

12 Die hierzu erstmals ausgewerteten württembergischen Akten Herzog Ulrichs und Herzog Christophs liegen im Staatsarchiv Marburg und ergänzen das von Seiler gezeichnete Bild. StA Marburg (künftig: StAM), Politisches Archiv des Landgrafen Philipp von Hessen (P. A.), Nr. 3082 und 3085.

${ }_{13} \mathrm{Zu}$ Philipp von Hessen vgl. Inge Auerbach (Hg.), Reformation und Landesherrschaft. Vorträge des Kongresses anlässlich des 500. Geburtstages des Landgrafen Philipp des Großmütigen von Hessen vom 10. bis 13. November 2004 in Marburg, Marburg 2005; Ursula Braasch-Schwersmann u.a. (Hg.), Landgraf Philipp der Großmütige 1504-1567. Hessen im Zentrum der Reform, Begleitband zu einer Ausstellung des Landes Hessen, Marburg/ 
stand, hatte der württembergische Herzog schon die Warnung empfangen, auf keinen Fall der Einsetzung Milchlings zuzustimmen, zumal der Pfälzer Kurfürst Friedrich sich ebenfalls gegen diesen Plan ausgesprochen habe und Pfalzgraf Heinrich als Fürstpropst mittlerweile ebenfalls am liebsten seine Zustimmung rückgängig machen wolle ${ }^{14}$. Mit dem Deutschmeister würde Württemberg einen schlechten Nachbarn bekommen, der vor allem den evangelischen Ständen höchst feindselig gesonnen sei.

Auch der Deutschmeister hatte seine Gesandten an den württembergischen Hof geschickt und dort für sein Anliegen werben lassen. Er hatte allerdings eine herbe Abfuhr hinnehmen müssen, besonders hinsichtlich der päpstlichen Verleihung. Ulrich ließ den Gesandten mitteilen, dass er als Schirmherr des Stifts die Unabhängigkeit, Privilegien und Freiheiten der Stiftsherren schützen werde und im Übrigen nicht daran denke, untüchtige Bullen des Papstes umzusetzen, der ohnehin nur wider die concordiam Germaniae nationis vorgehen wolle ${ }^{15}$. Doch wendete sich die politische Lage sehr schnell - zuungunsten der Ellwanger Stiftsherren und Württembergs.

Nach dem gewonnenen Schmalkaldischen Krieg sah sich Karl V. in die Lage versetzt, seinen Plan doch noch durchführen zu können. Damit hätte sich ein Sicherheitssystem katholischer Territorien an die Ostflanke des protestantischen Württemberg legen lassen und damit ein harter Sperrriegel gegen alle weiterführenden Pläne der protestantischen Stände. Die Absicht Karls muss wohl in seinen weit umfassenden Visionen wie Interim und kaiserlichem Bund zu einer Umgestaltung des Reiches eingeordnet werden, wobei ihm seine gewonnene Machtfülle dienen sollte ${ }^{16}$. Doch blieb die Furcht in Ellwangen vor einer Inkorporation lebendig. Dieser wollte man durch die rasche Wahl eines anderen mächtigen und einflussreichen Kandidaten entgegentreten, nachdem im Januar 1552 Propst Heinrich überraschend gestorben war.

Neustadt a.d. Aisch 2004; Wolfgang Breul/Holger Thomas GräF, Fürst, Reformation, Land - Aktuelle Forschungen zu Landgraf Philipp von Hessen (1504-1567), in: Archiv für Reformationsgeschichte 98 (2007) S.274-300; Volker PREss, Landgraf Philipp der Großmütige von Hessen, in: Klaus Scholder/Dieter Kleinmann (Hg.), Protestanten. Von Martin Luther bis Dietrich Bonhoeffer, Frankfurt am Main 21992, S.60-77; Gabriele Haug-Moritz, Reich und Konfessionsdissens im Reformationszeitalter. Überlegungen zur Reichskonfessionspolitik Landgraf Philipps des Großmütigen von Hessen, in: Hessisches Jahrbuch für Landesgeschichte 46 (1996) S.137-159; Walter HeInemeYer, Philipp der Großmütige und die Reformation in Hessen. Gesammelte Aufsätze zur hessischen Reformationsgeschichte, hg. von Hans-Peter Lachmann, Marburg 1997.

14 StAM P. A. 3082, fol.7, Landgraf Philipp an Herzog Ulrich, 13. Februar 1546.

15 StAM P.A. 3082, fol.13f., Herzog Ulrichs Antwort an die Gesandten des Deutschmeisters, 8. Februar 1546.

${ }^{16}$ Vgl. dazu Horst Rabe, Reichsbund und Interim. Die Verfassungs- und Religionspolitik Kaiser Karls V. und der Reichstag von Augsburg 1547/48, Köln/Wien 1971; Volker Press, Die Bundespläne Kaiser Karls V. und die Reichsverfassung, in: Ders., Das Alte Reich. Ausgewählte Aufsätze, hg. von Johannes Kunisch, Berlin 22000, S. 67-127. 


\section{[Die Abbildungen 1-4 können aus rechtlichen Gründen online nicht bereitgestellt werden.]}

Abb. 1: Fürstpropst Otto Truchsess von Waldburg (1553 - 1573), Äbtetafel in der Ellwanger Stiftskirche St. Vitus (Vorlage: Stadtarchiv Ellwangen).

Abb. 2: Fürstpropst Franz Ludwig von Pfalz-Neuburg (1694 - 1732), Äbtetafel in der Ellwanger Stiftskirche St. Vitus (Vorlage: Stadtarchiv Ellwangen).

Abb. 3: Fürstpropst Franz Georg von Schönborn (1732 - 1756), Äbtetafel in der Ellwanger Stiftskirche St. Vitus (Vorlage: Stadtarchiv Ellwangen).

Abb. 4: Ellwangen. Blick auf das historische Stadtzentrum mit der Stiftskirche St. Vitus und dem Kranz der Stiftsherrenhäuser, welche die Kirche halbkreisförmig umgeben (Vorlage: Stadtbauamt Ellwangen). 
Ungeachtet der kaiserlichen Protektion für Wolfgang Schutzbar schritt das Ellwanger Kapitel zur Wahl, aus der Otto Truchsess von Waldburg, Bischof von Augsburg, als Sieger hervorging (Abb.1 ${ }^{17}$. Diese Wahl war aus Ellwanger Sicht taktisch ein kluger Schritt. Denn der Kardinal hatte großen Einfluss bei Kaiser und Papst und verfügte in seinem Bistum über den notwendigen politischen und finanziellen Rückhalt. Seine konsequent altkirchliche und kaiserfreundliche Haltung stand außer jedem Zweifel.

Schnell sollte sich auch politisch das Blatt wieder wenden. Nach seiner Niederlage im Fürstenaufstand $1552^{18}$ konnte Karl V. endgültig nicht mehr zugunsten des Deutschmeisters eingreifen, der dennoch sein Vorhaben noch nicht aufgeben wollte. Die Auseinandersetzung hatte ein militärisches Nachspiel, weil Wolfgang Schutzbar am 4. Dezember 1552 still und heimlich während der Sonntagspredigt in Ellwangen mit Truppen einmarschierte und Besitz von der Fürstpropstei ergriff. Wiederum wandte sich das Stiftskapitel an seinen württembergischen Schutzherrn, nun an Herzog Christoph, der nach dem Sieg im Fürstenaufstand politisch wesentlich freier agieren konnte ${ }^{19}$. Auch Christoph sprach sein Vorgehen mit Landgraf Philipp von Hessen ab, indem er die frefenliche aigengwalltige und offenbare Land fridbruchige feindtliche handlung des Deutschmeisters anzeigte und ein energisches Vorgehen ankündigte, wofür er um Verständnis bat ${ }^{20}$. Landgraf Philipp hielt bezeichnenderweise eine solche Entschuldigung für reichlich überflüssig, dann wir denn Theutschen Meister unnd seyn stoltzen Kopff sebr woll kennen. ${ }^{21}$

$\mathrm{Zu}$ größeren militärischen Auseinandersetzungen kam es aber nicht mehr, denn als die württembergischen Truppen auf Ellwangen vorrückten, hatte Schutzbar bereits die Stadt verlassen. Herzog Christoph nahm die Deutschordensbesitzungen Horneck und Gundelsheim als Faustpfand zur Bezahlung seiner Kriegskosten ein, wobei diese Herrschaften auch das württembergische Territorium trefflich arron-

$17 \mathrm{Zu}$ Otto Truchsess von Waldburg vgl. Ferdinand Siebert, Zwischen Kaiser und Papst. Kardinal Truchseß von Waldburg und die Anfänge der Gegenreformation in Deutschland, Berlin 1943; Friedrich Zoepfl, Kardinal Otto Truchseß von Waldburg (1514-1573), in: Lebensbilder aus dem bayerischen Schwaben 4 (1955) S. 204-248; Thomas Groll/Walter Ansbacher (Hg.), Kardinal Otto Truchseß von Waldburg (1514-1573), Lindenberg 2015.

18 Franz Brendle, Um Erhalt und Ausbreitung des Evangeliums: Die Reformationskriege der deutschen Protestanten, in: Franz Brendle/Anton Schindling (Hg.), Religionskriege im Alten Reich und in Alteuropa, Münster ${ }^{2} 2010$, S.71-92.

$19 \mathrm{Zu}$ Herzog Christoph vgl. Hans-Martin Maurer, Herzog Christoph (1550-1568), in: Uhland, 900 Jahre Haus Württemberg (wie Anm. 11) S. 136-162; Franz Brendle, Christoph, in: Sönke Lorenz u.a. (Hg.), Das Haus Württemberg. Ein biographisches Lexikon, Stuttgart 1997, S. 108-111; Ders., Dynastie, Reich und Reformation (wie Anm. 11); Eberhard Fritz, Herzog Christoph von Württemberg, in: Hermle, Reformationsgeschichte Württembergs in Porträts (wie Anm. 11) S.199-225; Matthias Langensteiner, Für Land und Luthertum. Die Politik Herzog Christophs von Württemberg (1550-1568), Köln 2008.

20 StAM P.A. 3085, fol. 12, Herzog Christoph an Landgraf Philipp, 10. Dezember 1552.

21 StAM P. A. 3085, fol. 20, Landgraf Philipp an Herzog Christoph, 20. Dezember 1552. 
diert hätten. Unter Vermittlung des Würzburger Bischofs kam es dann zum Vergleich und zur Herausgabe der Herrschaften, allerdings nur unter Bezahlung einer hohen Entschädigungssumme für den Württemberger. Wolfgang Schutzbar strengte zwar noch einen Prozess vor der Rota an, den er aber 1562 verlor. Otto Truchsess von Waldburg blieb mit Hilfe Württembergs Fürstpropst von Ellwangen. Er war es dann auch, der Ellwangen fest im katholischen Lager verankerte und der 20 Jahre später den württembergischen Schutz und Schirm über das Stift ganz abschaffte $^{22}$. Er betrieb eine konsequente Politik der katholischen Reform auch mit Hilfe der Jesuiten, die er nach Ellwangen holte ${ }^{23}$. Der Augsburger Religionsfrieden von 1555 sorgte für die dafür nötige Sicherheit und bot mit dem geistlichen Vorbehalt - trotz dessen zweifelhafter Gültigkeit - doch einen gewissen Rechtsschutz ${ }^{24}$. Das Stift hatte seine Unabhängigkeit bewahrt und hat in der Folgezeit sehr selbstbewusst die Pröpste aus den eigenen Reihen gewählt, bis die ausgreifende Reichskirchenpolitik Kaiser Leopolds dem ein Ende setzte.

\section{Kaiserliche Kommissionen und kaiserliche Bitten}

Bei der Bildung kaiserlicher Kommissionen zur Untersuchung strittiger Angelegenheiten einzelner Reichsstände wurden regelmäßig geistliche Fürsten beteiligt. Ein bekanntes Beispiel hierfür sind die kaiserlichen Kommissionen zur Regelung der Verfassungsfragen in der freien Reichsstadt Frankfurt am Main während des Fettmilchaufstands am Anfang des 17. Jahrhunderts und während des Frankfurter Verfassungskonflikts im 18. Jahrhundert, bei denen jeweils Kurmainz neben Hessen-Darmstadt die Untersuchungen führte ${ }^{25}$. Nicht untersucht sind hingegen die zahlreichen Kommissionen, die der Fürstpropst von Ellwangen im Auftrag des Kaisers wahrnahm. Nach einer ersten Durchsicht der Akten hat es sich dabei eher um regionale Konflikte gehandelt als um die große Reichspolitik. Einige wenige seien hier genannt, um einen Eindruck zu vermitteln, mit welchen Fragen sich die

${ }^{22}$ Hermann Tüchle, Reformation und Gegenreformation im Gebiet der Fürstpropstei Ellwangen, in: BurR, Ellwangen 764-1964, Bd. 1 (wie Anm. 4) S.225-244.

${ }^{23}$ Dazu: Franz Brendle u.a. (Hg.), Jesuiten in Ellwangen. Oberdeutsche Provinz, Wallfahrt, Weltmission, Stuttgart 2012.

${ }^{24}$ Franz Brendel/Anton Schindling, Der Augsburger Religionsfrieden und die Germania Sacra, in: Carl A. Hoffmann u.a. (Hg.), Als Frieden möglich war. 450 Jahre Augsburger Religionsfrieden, Regensburg 2005, S.104-118.

25 Paul Hohenemser, Der Frankfurter Verfassungsstreit 1705-1732 und die kaiserlichen Kommissionen, Frankfurt a. M. 1920; Matthias Meyn, Die Reichsstadt Frankfurt vor dem Bürgeraufstand von 1612 bis 1614. Struktur und Krise, Frankfurt a. M. 1980; Anton ScHINDLING, Wachstum und Wandel vom Konfessionellen Zeitalter bis zum Zeitalter Ludwigs XIV. Frankfurt am Main 1555-1685, in: Frankfurt am Main. Die Geschichte der Stadt in neun Beiträgen, hg. von der Frankfurter Historischen Kommission, Sigmaringen ${ }^{2} 1994$, S. $205-260$. 
Ellwanger Kommissäre zu befassen hatten. Zahlreiche Erbschaftsstreitigkeiten etwa bei den Schenken von Limpurg ${ }^{26}$ oder den Fuggern zu Kirchberg und Weissenhorn $^{27}$, Rechtsstreitigkeiten zwischen dem Kloster Kaisheim und den Grafen von Öttingen ${ }^{28}$, der Streit um Obrigkeitsrechte zwischen den Rechbergern und der Gemeinde Böhmenkirch ${ }^{29}$, Auseinandersetzungen zwischen dem Stift Comburg und der Reichsstadt Rothenburg über das Vogteirecht zu Gebsattel ${ }^{30}$, Streitigkeiten um Geleitsrechte zwischen dem Herzog von Württemberg und der Reichsstadt Heilbronn $^{31}$, Steuerangelegenheiten zwischen Magistrat und Untertanen in der Reichsstadt Schwäbisch Gmünd ${ }^{32}$, um nur einige zu nennen.

In einem Fall wird Johann Jakob Blarer von Wartensee 1631 zu einer Debitkommission für den kaiserlichen Reichshofrat verordnet ${ }^{33}$. Debitkommissionen sollten verarmten Adligen bei der Bewältigung ihrer Schuldenkrise helfen, sie vor allem gegen bürgerliche Gläubiger schützen. In entsprechenden Fällen verordnete der Reichshofrat Kommissionen, die eine Bestandsaufnahme durchführten und nach Lösungsmöglichkeiten suchten ${ }^{34}$. Dabei wurde die kaiserliche Autorität zugunsten der Adligen ins Feld geführt, um eine Stundung, einen Nachlass oder eine Sequestration des Besitzes zu Händen des Kaisers zu erreichen. Gleichzeitig ging es aber auch darum, eine Veränderung der Wirtschaft- und Finanzführung durchzusetzen, um entsprechende Notsituationen oder gar den Bankrott in Zukunft zu vermeiden. Im Falle Blarers von Wartensee ging es um die Entschuldung der Erben des Hans Ludwig von Knöringen aus einer Reichsritterfamilie, die in den Kantonen Kocher und Altmühl immatrikuliert war und mit Heinrich von Knöringen den Augsburger Bischof im Dreißigjährigen Krieg stellte.

Der Westfälische Friede brachte neue Aufgaben für kaiserliche Kommissionen, die sich vor allem um die Ausführungsbestimmungen in der Religionsfrage dreh-

26 StAL B 397 II Bü17-Bü 20.

27 StAL B 397 II Bü 1448, Bü 1449.

28 StAL B 397 II Bü 51-Bü 56.

29 StAL B 397 II Bü 1359, Bü 1360.

30 StAL B 397 II Bü 1354-Bü 1358.

31 StAL B 397 II Bü 1409.

32 StAL B 397 II Bü 102.

33 StAL B 397 II Bü 1406.

${ }^{34} \mathrm{Zu}$ den Debitkommissionen vgl. Volker Press, Die aufgeschobene Mediatisierung. Finanzkrise der Kleinstaaten und kaiserliche Stabilisierungspolitik, in: 32. Versammlung deutscher Historiker in Hamburg (Beiheft zu Geschichte in Wissenschaft und Unterricht), Stuttgart 1979, S.139-141; Susanne Hermann, Die Durchführung von Schuldenverfahren im Rahmen kaiserlicher Debitkommissionen im 18. Jahrhundert am Beispiel des Debitwesens der Grafen von Montfort, in: Wolfgang Sellert (Hg.), Reichshofrat und Reichskammergericht. Ein Konkurrenzverhältnis, Köln u.a. 1999, S.111-127; Jürgen Ackermann, Verschuldung, Reichsdebitverwaltung, Mediatisierung. Eine Studie zu den Finanzproblemen der mindermächtigen Stände im alten Reich. Das Beispiel der Grafschaft Ysenburg-Büdingen 1687-1806, Marburg 2002. Nach wie vor unverzichtbar: Johann Jacob Moser, Von dem Reichs-Ständischen Schuldenwesen, 2 Bde., Frankfurt/Leipzig 1774. 
ten, etwa um Streitigkeiten zwischen dem Kloster Kirchheim am Ries und dem Grafen von Oettingen-Oettingen wegen Störung der Religionsausübung ${ }^{35}$. Besonderes Augenmerk erforderten die konfessionellen Verhältnisse in den bikonfessionellen Reichsstädten in Schwaben, in denen die Parität durch den Westfälischen Frieden festgelegt worden war, wobei die genaue Durchführung doch immer wieder Schwierigkeiten bereitete. Der Reichshofrat achtete peinlich darauf, dass jeweils sowohl ein katholischer als auch ein protestantischer Vertreter als Schlichter auftraten. So wurden der Ellwanger Fürstpropst und die evangelische Reichsstadt Schwäbisch Hall zu Kommissaren in den Auseinandersetzungen zwischen den protestantischen Räten der Reichsstadt Ravensburg und dem dortigen katholischen Magistrat eingesetzt, die sich über die Modalitäten der Ratswahl und andere Gravamina nicht einigen konnten ${ }^{36}$.

Das Recht der Ersten Bitten, das ius primae precis, geht bis ins 13. Jahrhundert zurück $^{37}$. Seit dieser Zeit konnten die neugewählten Könige oder Kaiser an allen Dom- und Kollegiatstiften das nächste frei werdende Kanonikat besetzen. Damit war es ihnen möglich, in die Zusammensetzung der Domkapitel einzugreifen. Nur wenigen großen Domstiften gelang es, dieses Recht einzuschränken. Der ausgeprägte Machtwille der Domherren und ihr Streben nach Unabhängigkeit und Eigenständigkeit stießen hier doch an gewisse Grenzen ${ }^{38}$. Auch in Ellwangen machten die deutschen Könige, mit Ausnahme Ferdinands I., von diesem Recht Gebrauch und waren damit äußerst erfolgreich. Denn die Ellwanger Stiftsherrn respektierten oft die kaiserlichen Bitten, sofern der Kandidat nicht gänzlich ungeeignet war oder eigene Interessen des Stiftskapitels dominierten.

Neben einzelnen Precisten, die den Familien des Ellwanger Stiftsadels angehörten, wie den Freiberg und Rechberg, stammten die meisten Bewerber aus Familien, die in Diensten des Wiener Hofes und der Habsburger Vorlande standen oder die Beziehungen zum Hof hatten ${ }^{39}$. Familien wie die Daun, die von Ulm oder die Pfirt hatten sich über Jahrzehnte hinweg als treue Parteigänger der Habsburger erwiesen und konnten nun eine Gegenleistung dafür einfordern. Elf erste Bitten des Kaisers wurden erfüllt, neun weitere Precisten wurden in Ellwangen nicht akzeptiert, sei

35 StAL B 397 II Bü 91.

36 StAL B 397 II Bü 1411.

37 Hans Erich Feine, Papst, Erste Bitten und Regierungsantritt des Kaisers seit dem Ausgang des Mittelalters, in: Zeitschrift für Rechtsgeschichte Kan. Abt. 20 (1931) S.1-101.

38 Zur Rolle der Domkapitel vgl. Günter CHRIst, Selbstverständnis und Rolle der Domkapitel in den geistlichen Territorien des alten deutschen Reiches in der Frühneuzeit, in: Zeitschrift für Historische Forschung 16 (1989) S. 257-328; Franz Brendle, Die Domkapitel als Conregentes der Fürstbischöfe in den geistlichen Staaten, in: Gerhard Ammerer u. a. (Hg.), Höfe und Residenzen geistlicher Fürsten. Strukturen, Regionen und Salzburgs Beispiel in Mittelalter und Neuzeit, Ergebnisse der internationalen und interdisziplinären Tagung in der Salzburger Residenz 19.-22. Februar 2009, Sigmaringen 2010, S. 93-107.

$39 \mathrm{Zu}$ den kaiserlichen primae preces in Ellwangen vgl. Eduard Mildner, Das Ellwanger Stiftskapitel in seiner persönlichen Zusammensetzung, Tübingen 1969, S. 47 ff. 
es, weil sie die kanonischen Voraussetzungen nicht erfüllten, sei es, weil das Ellwanger Kapitel eigenen Kandidaten verpflichtet war. Die Ablehnungsgründe waren nicht immer so offensichtlich wie im Falle von Paul Prugkschlegel. Hier hatte Maximilian II. den Sohn seines Mundkochs vorgeschlagen, was vom Ellwanger Kapitel aber abgelehnt wurde. Dennoch: mit den kaiserlichen Ersten Bitten ebenso wie mit päpstlichen Provisionen erweiterte sich das Herkunftsfeld der Stiftskapitulare. Das Ellwanger Stift wurde dadurch auch besser in der Reichspolitik integriert und Teil des sich entfaltenden Reichskirchensystems. Das kam der seit den 1660er Jahren ausgreifenden kaiserlichen Politik zugute, die unter Leopold I. eine neue Dynamik entfaltete.

\section{Der Kaiser und die Ellwanger Propstwahlen}

Kaiser Leopold I. (1658-1705) gilt als der Begründer der österreichischen Donaumonarchie, der den habsburgischen Herrschaftsbereich beträchtlich erweiterte $^{40}$. Unter seiner Herrschaft vollzog sich der Aufstieg der Monarchie zu einer europäischen Großmacht. Die Abwehrsiege gegen Franzosen und Türken ließen das habsburgische Kaisertum noch einmal in altem Glanz erstrahlen und führten Leopold I. in den Zenit seiner Macht. Gleichzeitig stand seine Regierungszeit auch im Zeichen einer erneuerten Reichspolitik, die dem habsburgischen Kaisertum eine herausragende Stellung verschaffte, einer barocken Umgestaltung des Reiches Vorschub leistete und auch die Reichskirche zu einer neuen Blüte führte ${ }^{41}$. Leopold I. gelang es, die kaiserliche Klientel der kleinen und mindermächtigen Fürsten und Städte wieder stärker an das Reichsoberhaupt zu binden ${ }^{42}$. Der Kaiser baute sein Gesandtschaftswesen konsequent aus und sorgte damit für enge diplomatische Verbindungen zu den Höfen und Herrschaftszentren im Reich ${ }^{43}$. Ebenso wie der Reichstag in Regensburg zunehmend zu einem Instrument kaiserlicher Reichspolitik wurde, setzte der Reichshofrat als politisches Beratungsgremium und als

${ }^{40} \mathrm{Zu}$ Leopold I. vgl. Franz Brendle, Das habsburgische Herrscherhaus: Die Kaiser Leopold I., Joseph I. und Karl VI., in: Donat Büchel/Rainer Vollkommer (Hg.), 1712 das Werden eines Landes, Vaduz 2012, S. 85-95; John P. Spielman, Leopold I. Zur Macht nicht geboren, Graz u. a. 1981.

${ }^{41}$ Zur erneuerten Reichspolitik Leopolds I. vgl. Volker Press, Die kaiserliche Stellung im Reich zwischen 1648 und 1740. Versuch einer Neubewertung, in: Ders., Das Alte Reich (wie Anm. 16) S.189-222.

42 Volker Press, Patronat und Klientel im Heiligen Römischen Reich, in: Antoni MaçZaK (Hg.), Klientelsysteme im Europa der Frühen Neuzeit, München 1988, S. 19-46; Berthold SutTER, Kaisertreue oder rationale Überlebensstrategie? Die Reichsritterschaft als habsburgische Klientel im Reich, in: Heinz Duchhardt/Matthias Schnettger (Hg.), Reichsständische Libertät und habsburgisches Kaisertum, Mainz 1999, S. 257-308.

${ }^{43}$ Volker Press, The Habsburg Court as Center of the Imperial Government, in: The Journal of Modern History 58/Supplement 1986, S.23-45. 
oberstes Reichsgericht effektiv Akzente ${ }^{44}$. Kaiserliche Debitkommissionen, wie die bereits erwähnte zur Schuldenregulierung, wurden von Wien in die Territorien entsandt, um dort überschuldete Territorialherren vor ihren überwiegend bürgerlichen und jüdischen Gläubigern zu schützen.

Aber auch in der Reichskirche war das Reichsoberhaupt durch seine Kommissare bei Bischofs- und Abtswahlen ständig präsent ${ }^{45}$. Mangels eigener Kandidaten übten hier verwandte und befreundete Dynastien wie die Pfalz-Neuburger, die Lothringer oder die Schönborn eine Art Stellvertreterfunktion für das Kaiserhaus $\mathrm{aus}^{46}$. Gerade die geistlichen Staaten im Reich entwickelten durch ihre katholische Ausrichtung und barocke Ausgestaltung eine besondere Affinität zur habsburgischen Herrscherdynastie und zum Wiener Hof ${ }^{47}$. Hier wurden Karrieren in Gesellschaft, in Verwaltung und Armee vorbereitet, hier entwickelte sich eine Anlaufstelle für viele Adlige aus dem Reich und aus den österreichischen Erblanden ${ }^{48}$. Diese erneuerte kaiserliche Reichspolitik hatte auch Auswirkungen auf die Fürstpropstei Ellwangen.

Bis 1689 war die Fürstpropstei eine Domäne des niederen Adels, insbesondere gestützt auf die Reichsritterschaft am Kocher ${ }^{49}$. Aus dem Kapitel selbst gingen 13 der 20 Fürstpröpste hervor. Dies änderte sich nun mit dem Einbezug Ellwangens in die große Reichskirchenpolitik des Kaisers und der katholischen Dynastien ${ }^{50}$.

${ }^{44}$ Volker Press, Der Reichshofrat im System des frühneuzeitlichen Reiches, in: Friedrich Battenberg/Filippo Ranieri (Hg.), Geschichte der Zentraljustiz in Mitteleuropa, Festschrift für Bernhard Diestelkamp zum 65. Geburtstag, Weimar u.a. 1994, S.349-363.

${ }^{45}$ Vgl. dazu Günter Christ, Praesentia Regis. Kaiserliche Diplomatie und Reichskirchenpolitik vornehmlich am Beispiel der Entwicklung des Zeremoniells für die kaiserlichen Wahlgesandten in Würzburg und Bamberg, Wiesbaden 1975; Matthias SchnetTger, Der Kaiser und die Bischofswahlen. Das Haus Österreich und die Reichskirche vom Augsburger Religionsfrieden bis zur Mitte des 17. Jahrhunderts, in: DuchHardt/Schnettger, Reichsständische Libertät (wie Anm. 42) S.213-256.

46 Volker Press, Fürstentum und Fürstenhaus Pfalz-Neuburg. Die dritte wittelsbachische Kraft, in: Konrad AcKermann/Georg Girisch (Hg.), Gustl Lang. Ein Leben für die Heimat, Weiden 1989, S.255-278; Hubert Wolf, Die Reichskirchenpolitik des Hauses Lothringen (1680-1715). Eine Habsburger Sekundogenitur im Reich?, Stuttgart 1994.

${ }^{47}$ Hubert Jedin, Die Reichskirche der Schönbornzeit, in: Trierer theologische Zeitschrift 65 (1956) S. 202-216; Ludwig HüTTL, Geistlicher Fürst und geistliche Fürstentümer im Barock und Rokoko, in: Zeitschrift für bayerische Landesgeschichte 37 (1974) S.3-48.

${ }^{48}$ Franz Brendle/Anton Schindling, Reichskirche und Reich in der Frühen Neuzeit, in: Volker Himmelein/Hans Ulrich Rudolf, Alte Klöster, Neue Herren. Die Säkularisation im Deutschen Südwesten 1803, Bd.2.1, Ostfildern 2003, S.3-22; Franz BRENdLE/ Anton Schindling, Germania sacra - Reichskirche, in: Stephan Wendehorst/Siegrid Westphal (Hg.), Lesebuch Altes Reich, München 2006, S.211-215.

49 Thomas Schulz, Das Fürststift Ellwangen und die Ritterschaft am Kocher, in: Ellwanger Jahrbuch 31 (1985/86) S. 44-52.

50 Vgl. dazu Rudolf ReinHardt, Untersuchungen zur Besetzung der Propstei Ellwangen seit dem 16. Jahrhundert. Zugleich ein Beitrag zur politischen Geschichte des Stifts, in: Burr, Ellwangen 764-1964, Bd.1 (wie Anm.4) S.316-378; Franz Brendle, Die geistlichen 
Die hochadeligen Familien der Pfalz-Neuburg, Schönborn und Sachsen betrachteten das Fürststift als eine einträgliche Nebenpfründe. Auch das Kapitel selbst begann sich nun stärker auf den Hochadel hin umzustrukturieren. Während Fürstpröpste aus dem niederen Adel meist auf andere Kollegiatspfründen verzichten mussten, konnten hochadelige Inhaber aufgrund von Dispensen verschiedene Ämter und Pfründen mit Ellwangen kombinieren ${ }^{51}$. Die geforderte Residenzpflicht wurde in diesen Fällen kaum wahrgenommen ${ }^{52}$. Die auswärts residierenden Pröpste bestellten meist den Dekan zu ihrem Statthalter und betrauten ihn mit den Regierungsgeschäften.

Seit der Mitte des 17. Jahrhunderts standen dem habsburgischen Erzhaus keine Kandidaten mehr für eine ausgreifende Reichskirchenpolitik zur Verfügung. Leopold I. war ja selbst für eine Karriere in der Germania Sacra vorgesehen gewesen, bevor er nach dem Tod Ferdinands IV. die Nachfolge im Kaisertum antrat. Aus diesem Umstand erwuchs eine Art Ersatzrolle zunächst für das Haus Pfalz-Neuburg $^{53}$. Es gab fast kein Hochstift im Reich, um das sich die Familie nicht in der Zeit von 1680 bis 1720 bemüht hätte. Nach ihrer Konversion zum Katholizismus setzten die Neuburger, die seit 1685 Kurfürsten von der Pfalz waren, verstärkt auf den Wiener Hof. Die Koalition wurde durch die Ehe Kaiser Leopolds mit Eleonore von Pfalz-Neuburg besiegelt, die sich fortan in Wien für die Ziele und Interessen der pfälzischen Dynastie einsetzte, insbesondere für ihre Brüder Ludwig Anton und Franz Ludwig, die beide beachtliche Karrieren in der Reichskirche machen sollten.

Im engen Zusammenspiel mit Wien traten 1689 die Pfalz-Neuburger auch in Ellwangen auf den Plan. Neben den großen Hochstiften des Reiches geriet die Fürstpropstei in den Blickpunkt der Pfälzer, bot das Stift doch die Gelegenheit, die finanziellen Einkünfte aufzubessern, was für sie durch den Krieg im Westen dringend notwendig war. In einer engen Aktionseinheit mit Wien wurden die Interessen durchgefochten: Ellwangen sollte als Glied der Reichskirche in die

Reichsstände Schwabens im System der Germania Sacra, in: Wolfgang Wüst u.a. (Hg.), Grenzüberschreitungen. Die Außenbeziehungen Schwabens in Mittelalter und Neuzeit, Augsburg 2008, S. 47-58.

${ }^{51}$ Zum Problem der Kumulation vgl. Rudolf Reinhardt, Die Kumulation von Kirchenämtern in der deutschen Kirche der frühen Neuzeit, in: Ders., Reich - Kirche Politik. Ausgewählte Beiträge zur Geschichte der Germania Sacra in der Frühen Neuzeit, Ostfildern 1998, S. 204-222; Wolfgang Wüst, Personalunionen zwischen Stiftsstaaten. Administrative Chance oder Regierungschaos?, in: Ders. (Hg.), Geistliche Staaten in Oberdeutschland im Rahmen der Reichsverfassung. Kultur - Verfassung - Wirtschaft Gesellschaft. Ansätze zu einer Neubewertung, Epfendorf 2002, S.163-186.

52 Zum Bild der geistlichen Fürsten vgl. Wolfgang Wüst, Bischöfe als Reichsfürsten. Wahlverpflichtungen, Machtbarrieren, Überforderung und Vielregiererei in süddeutschen Hochstiften, in: Bettina Braun u.a. (Hg.), Geistliche Fürsten und Geistliche Staaten in der Spätphase des Alten Reiches, Epfendorf 2008, S.43-60.

${ }_{53}$ Rudolf Reinhardt, Zur Reichskirchenpolitik der Pfalz-Neuburger Dynastie, in: Historisches Jahrbuch 84 (1964) S. 118-128. 
kaiserliche Klientel eingebunden werden. Kandidaten für die Propstwürde waren 1689 die beiden Brüder Ludwig Anton und Alexander Sigismund ${ }^{54}$, die sich schon zuvor um eine Koadjutorie, allerdings ohne Erfolg, bemüht hatten. Beide waren Söhne des regierenden Kurfürsten Philipp Wilhelm von der Pfalz und schienen als unmittelbare Nachbarn des Stifts (Ludwig Anton war Hoch- und Deutschmeister, Alexander Sigismund Koadjutor in Augsburg) am ehesten in Frage zu kommen.

Die Ausgangslage des Deutschmeisters war weitaus günstiger, hatte er doch eine starke Partei im Kapitel hinter sich sowie ein päpstliches Breve elegibilitatis, so dass für seine Postulation nur die einfache Stimmenmehrheit notwendig war ${ }^{55} . \mathrm{Da}$ ihn sein Bruder, der offenbar selbst nur geringe Absichten hatte, von Anfang an unterstützte, und auch das kaiserliche Empfehlungsschreiben, obwohl für beide Kandidaten ein solches ausgefertigt wurde, unmissverständlich klar machte, dass der Kaiser eine Wahl in seinem Sinne erwartete, blieb dem Stiftskapitel wenig Handlungsspielraum. Es beugte sich dem Druck und wählte am 2. August 1689 Ludwig Anton von Pfalz-Neuburg zum neuen Fürstpropst, wenn auch nicht einstimmig ${ }^{56}$. Während der Wahl hielt sich Leopold nicht nur demonstrativ im nahen Neuburg auf, am Wahltag selbst war Heinrich Theobald von Goldstein als Gesandter des Kaisers erschienen, der offen und unverblümt eine Wahl im Sinne des Kaisers verlangte. Leopold erwarte vom Kapitel, dass sein Vorschlag in gewierige Reflexion gezogen würde, da er für das Stift nur von Vorteil sein könne. Der Deutschmeister wurde per maiora, jedoch nicht wie vom Kaiser gewünscht, mit einhelligen vota gewählt ${ }^{57}$. Damit war die alte Kombination, die schon Karl V. 1552 im Auge gehabt hatte, hergestellt: die Verbindung Ellwangens mit dem Deutschordensland um Mergentheim. Doch hatten sich die Autonomie des Stifts und seine Stellung als unabhängiger Reichsstand so sehr gefestigt, dass eine Einverleibung in das Deutschordensgebiet nicht mehr zur Disposition stand.

Der Bruder des Pfälzers, Franz Ludwig von Pfalz-Neuburg, wurde nach dem frühen Tod Ludwig Antons 1694 problemlos zum neuen Fürstpropst gewählt $\left(\mathrm{Abb} .{ }^{2}\right)^{58}$. Eine kaiserliche Wahlgesandtschaft war erst gar nicht erschienen, da

${ }^{54} \mathrm{Zu}$ Alexander Sigismund von Pfalz-Neuburg vgl. Josef Johannes Schmid, Alexander Sigismund von Pfalz-Neuburg, Fürstbischof von Augsburg 1690-1737. Ein Beitrag zur Kulturgeschichte Schwabens im Hochbarock, Weißenhorn 1999.

${ }^{55}$ Vgl. zu diesen Vorgängen ReinHardt, Untersuchungen zur Besetzung (wie Anm. 50) S. $340 \mathrm{ff}$.

$56 \mathrm{Zu}$ Ludwig Anton von Pfalz-Neuburg vgl. Maria Lehner, Ludwig Anton von PfalzNeuburg (1660-1694), Marburg 1994.

57 Zitate nach ReinHARdt, Untersuchungen zur Besetzung (wie Anm. 50) S. $343 \mathrm{f}$.

$58 \mathrm{Zu}$ Franz Ludwig von Pfalz-Neuburg vgl. Johannes HiLs, Das Wirken des Kurfürsten Franz Ludwig, Pfalzgrafen bei Rhein, als Fürstbischof von Breslau, in: Ellwanger Jahrbuch 27 (1977/1978) S.162-167; Bernhard Demel, Franz Ludwig von Pfalz-Neuburg als Hoch- und Deutschmeister (1694-1732) und Bischof von Breslau (1683-1732), in: Jahrbuch der Schlesischen Friedrich-Wilhelms-Universität zu Breslau 36/37 (1995/1996) S. 93-150; Hubert Wolf, Menschenfischer - Pfründenjäger. Franz Ludwig von Pfalz-Neu- 
man sich wohl der Wahl sicher glaubte. Dem Kapitel in Ellwangen muss ohnehin klar gewesen sein, wer hinter dem Pfalz-Neuburger stand. So kam Franz Ludwig mit seiner Selbsteinschätzung der Wahrheit sicher nahe, wenn er argwöhnte, seine Wahl basiere auf einem Effect und Zuwurff dero Kaiserlichen Hulden ${ }^{59}$. Leopold I. wies jegliche Einmischung Wiens allerdings kategorisch zurück: Die Wahl sei erfolgt ohne sonderbare menschliche Cooperation durch göttliche Eingebung ${ }^{60}$. Dennoch: Die Karriere Franz Ludwigs in der Reichskirche als Bischof von Breslau und Worms, Hoch- und Deutschmeister des Deutschen Ordens, Fürstpropst von Ellwangen und Kurfürst-Erzbischof von Trier und - nach Verzicht auf Trier - Kurfürst-Erzbischof von Mainz, wäre ohne die Unterstützung des kaiserlichen Hofes kaum möglich gewesen. Trotz des schnellen Erfolgs des Pfalz-Neuburgers werden auch bei diesem Wahlakt deutlich die Bedenken des Ellwanger Stiftskapitels deutlich, das wiederum zu keinem einstimmigen Ergebnis, sondern lediglich zu einer Mehrheitsentscheidung gekommen war. Doch hatten es wohl die schwierigen Zeitumstände angeraten sein lassen, weiter auf das kaiserliche Wohlwollen zu setzen. Schließlich dauerte der Krieg im Westen noch an und war ein Friede mit Frankreich noch nicht geschlossen. Da mussten die Ambitionen des einen oder anderen Stiftsherrn, selbst Propst werden zu können, wohl oder übel zurückstehen. Doch werden hier die Grenzen der kaiserlichen Einflussnahme ebenso deutlich wie im Vorgehen des Stiftskapitels im Bezug auf die Wahlkapitulationen. Seit Otto von Waldburg war bei jeder Wahl eine solche abgeschlossen worden, um die Rechte des Kapitels festzuhalten ${ }^{61}$. Entgegen des kaiserlichen und päpstlichen Verbots von 1698 hielten die Kapitulare - wie auch andernorts - bis zum Ende des Alten Reiches an dieser Praxis fest ${ }^{62}$.

Das Erbe der Neuburger als Hauptexponent der kaiserlichen Reichskirchenpolitik trat das Haus Schönborn an, das durch die Unterstützung des Kaisers und ein gegenseitiges Protektionssystem zur erfolgreichsten Bischofsdynastie der Neuzeit neben den Wittelsbachern avancierte ${ }^{63}$. Sofort nach dem Tod Franz Ludwigs

burg, die Reichskirche und Ellwangen, in: Ellwanger Jahrbuch 37 (1997/1998) S.15-38; Bernhard Demel, Franz Ludwig von Pfalz-Neuburg, in: Udo Arnold (Hg.), Die Hochmeister des Deutschen Ordens 1190-1994, Marburg 1998, S. 239-247.

59 Zitiert nach ReInHARDT, Untersuchungen zur Besetzung (wie Anm. 50) S. 347.

60 Zitiert nach ebd.

${ }^{61}$ Franz Brendle, Die Wahlkapitulationen der Ellwanger Fürstpröpste, in: Ellwanger Jahrbuch 33 (1989/90) S.76-120.

${ }^{62}$ Rudolf Vierhaus, Wahlkapitulationen in den geistlichen Staaten des Reiches im 18. Jahrhundert, in: Ders. (Hg.), Herrschaftsverträge, Wahlkapitulationen, Fundamentalgesetze, Göttingen 1977, S. 205-219.

63 Alfred Schröcker, Ein Schönborn im Reich. Studien zur Reichspolitik des Fürstbischofs Lothar Franz von Schönborn (1655-1729), Wiesbaden 1978; Ders., Die Patronage des Lothar Franz von Schönborn (1655-1729). Sozialgeschichtliche Studie zum Beziehungsnetz in der Germania Sacra, Wiesbaden 1981; Sylvia Schraut, Das Haus Schönborn. Eine Familienbiographie. Katholischer Reichsadel 1640-1840, Paderborn u. a. 2005. 
teilte das Ellwanger Stiftskapitel dem Kaiserhof die Nachricht in einem Notifikationsschreiben mit, ohne allerdings den Wahltermin anzuzeigen. Man wusste ihn zu dieser Zeit offenbar selbst noch nicht; dennoch erregte dieser Umstand am kaiserlichen Hof erhebliches Missfallen. Karl VI. hatte nämlich die Absicht, als römischer Kayser, Obrister Schutzvogt der christlichen catholischen Kirchen und höchster Lehensherr die Neuwahl durch die Anwesenheit eines Gesandten zu beehren ${ }^{64}$. Dabei wurde ausdrücklich auf die anderen geistlichen Staaten des Reichs verwiesen, wo ein solches Vorgehen ebenfalls üblich sei. Entsprechend habe auch das Ellwanger Stiftskapitel Kaiser und Reich die schuldige Pflicht zu leisten.

Gleich drei Kandidaten des Hauses Schönborn bewarben sich 1732 um Ellwangen: Damian Hugo, Kardinal-Bischof von Speyer ${ }^{65}$, Friedrich Karl, Bischof von Würzburg und Bamberg ${ }^{66}$, und Franz Georg, Erzbischof von Trier (Abb.3). Die kaiserliche Politik richtete sich vor allem gegen die ausgreifende bayerische Politik und damit gegen den Kandidaten Bayerns, Kardinal Johann Theodor, Bischof von Freising und Regensburg. Durch ihre reichsritterschaftliche Abkunft übten die Schönborn eine nicht zu unterschätzende Attraktivität auf die Ellwanger Stiftsherren aus, andererseits konnte das Kapitel keinen eigenen Kapitular präsentieren, der die Mehrheit des Kapitels hinter sich hätte bringen können. Lange Zeit war die Situation so unübersichtlich, dass der Stiftsherr von Spaur die Ansicht äußerte, nur der liebe Gott wisse, wer in Ellwangen gewählt werde ${ }^{67}$. Letztlich setzte sich das Werben Karls VI. auch bei den Kapitularen durch, die dem Kaiserhaus und seinen Kandidaten eher reservierend gegenüber standen. Franz Georg von Schönborn wurde einstimmig gewählt, nachdem seine Brüder aus Familiensolidarität zurückgewichen waren ${ }^{68}$.

Nachdem 1756 mit Anton Ignaz Fugger ein relativ farbloser Kandidat aus den eigenen Reihen gewählt werden konnte, konnte über das Instrument der Koadjutorie mit Clemens Wenzeslaus von Sachsen erneut ein habsburgischer Kandidat als letzter Fürstpropst von Ellwangen - sein Amt antreten ${ }^{69}$. Da die sächsischen

${ }^{64}$ Zitiert nach Reinhardt, Untersuchungen zur Besetzung (wie Anm. 50) S. 348.

65 Stephan Mauelshagen, Ordensritter - Landesherr - Kirchenfürst: Damian Hugo von Schönborn (1676-1743). Ein Leben im alten Reich, Ubstadt-Weiher 2001.

${ }^{66}$ Karl Wild, Staat und Wirtschaft in den Bistümern Würzburg und Bamberg. Eine Untersuchung über die organisatorische Tätigkeit des Bischofs Friedrich Karl von Schönborn 1729-1746, Heidelberg 1906; Hugo Hantsch, Reichsvizekanzler Friedrich Karl, Graf von Schönborn (1674-1746). Einige Kapitel zur politischen Geschichte Kaiser Josefs I. und Karls VI., Augsburg 1929.

67 Reinhardt, Untersuchungen zur Besetzung (wie Anm. 50) S.350.

$68 \mathrm{Zu}$ Franz Georg von Schönborn vgl. Franz Zierlein, Franz Georg Graf von Schönborn 1682-1756, in: Ellwanger Jahrbuch 23 (1969/1970) S.79-116; Hans Pfeifer, Aufklärung und Absolutismus in der Fürstpropstei Ellwangen, in: Ellwanger Jahrbuch 19 (1960/ 1961) S. $63-83$.

69 Zum Instrument der Koadjutorie Rudolf ReInHardt, Die Koadjutorie mit dem Recht der Nachfolge in der neuzeitlichen Reichskirche, in: Ellwanger Jahrbuch 31 (1985/86) S. $13-43$. 
Wettiner anlässlich der polnischen Königswahl Kurfürst Augusts des Starken katholisch geworden waren, konnte Clemens Wenzeslaus drei Generationen später eine reichskirchliche Karriere beginnen, die ganz im Zeichen der Annäherung zwischen Habsburg und Sachsen im Siebenjährigen Krieg stand. Als Sohn einer Habsburgerin, zudem verwandt und verschwägert mit den bayerischen Wittelsbachern, eröffneten sich dem sächsischen Prinzen glänzende Aussichten, die ihm schließlich zu den reichskirchlichen Würden von Trier, Augsburg und Ellwangen verhalfen ${ }^{70}$.

\section{Der Kaiser und das Ende der Fürstpropstei}

1770 hatte Anton Ignaz Fugger den Trierer Kurfürsten zum Koadjutor angenommen und erhielt dafür im Tausch das Bistum Regensburg ${ }^{71}$. Bis zu seinem Tod 1787 war Fugger hauptsächlich in seinem Bistum, 1777 übernahm Clemens Wenzeslaus die Regierungsgeschäfte in Ellwangen, bis das Stift in der napoleonischen Flurbereinigung seine Selbständigkeit verlor. Bis zuletzt hatte man in Ellwangen auf den Schutz von Kaiser und Reich vertraut, obwohl sich Konturen des Untergangs bereits abzeichneten. Ein signifikantes Beispiel für die Anhänglichkeit an das habsburgische Herrscherhaus ist der Ellwanger Regierungsrat Joseph von Sartori. Er ist in erster Linie dadurch bekannt geworden, dass er das berühmte Preisausschreiben des Fuldaer Domherrn von Bibra ${ }^{72}$ zur Zukunft der geistlichen Staaten mit einer Abhandlung gewann, die den dringenden Reformbedarf der Reichskirche

70 Anton Gulielminetti, Klemens Wenzeslaus. Der letzte Fürstbischof von Augsburg und die religiös-kirchliche Reformbewegung, in: Archiv für die Geschichte des Hochstifts Augsburg 1 (1909) S. 493-598; Hildebrand Troll, Kurfürst Klemens Wenzeslaus, Fürstbischof von Augsburg, in: Lebensbilder aus dem Bayerischen Schwaben 2, München 1953, S.302-325; Heribert RAAB, Clemens Wenzeslaus von Sachsen und seine Zeit 1739-1812, Bd.1: Dynastie, Kirche und Reich im 18. Jahrhundert, Freiburg u. a. 1962; Wolfgang WüsT, Fürstbischöfliche Amts- und Staatsführung im Hochstift Augsburg unter Clemens Wenzeslaus (1768-1803), in: Pankraz Fried, Miscellanea Suevica Augustana. Der Stadt Augsburg dargebracht zur 2000-Jahrfeier 1985, Sigmaringen 1985, S.129-146; Peter Rummel, Kurfürst Klemens Wenzeslaus und sein Augsburger Generalvikar Franz Heinrich Beck, in: Jahrbuch des Vereins für Augsburger Bistumsgeschichte 22 (1988) S.75-104; Theodor Rolle, Fürstbischof Clemens Wenzeslaus und Kurfürst Max IV./König Max I. Joseph von Bayern. Zu den Auseinandersetzungen über das Verhältnis von Kirche und Staat in Bayern in den Jahren 1802-1806, in: Jahrbuch des Vereins für Augsburger Bistumsgeschichte 25 (1991) S. 109-142; Gabriele B. Clemens, Clemens Wenzeslaus von Sachsen (1739-1812), in: Elisabeth DüHR (Hg.), Unter der Trikolore. Sous le drapeau tricolore. Trier in Frankreich, Napoleon in Trier, 1794-1814. Ausstellung 6. Juni-31. Oktober 2004, Katalog-Handbuch, Trier 2004, S. 95-103.

71 Erhard Meissner, Fürstbischof Anton Ignaz Fugger (1711-1787), Tübingen 1969.

72 Vgl. dazu Max Braubach, Die kirchliche Aufklärung im katholischen Deutschland im Spiegel des Journal von und für Deutschland, in: Historisches Jahrbuch 54 (1934) S.1-63. 
zwar anmahnte, aber dennoch ihre Existenzberechtigung betonte ${ }^{73}$. Nach seiner Tätigkeit in Ellwangen übersiedelte er nach Wien, wo er sich seit 1789 in der Umgebung Josephs II. aufhielt und 1792/93 die „Leopoldinische[n] Annalen. Ein Beitrag zur Regierungsgeschichte Kaiser Leopolds II.“ verfasste ${ }^{74}$. In diesem Werk geht er auch auf Joseph II. ein und gibt eine knappe Darstellung über die Anfangszeit der Regierung Franz’ II. Zu dieser Zeit war er bereits „Kaiserlicher Rat“ im Dienste Leopolds II. Diesen Titel hatte er noch, als die „Memoiren über die wichtigsten Staats-Materien unserer Zeit“ in den Jahren 1795 und 1797 veröffentlicht wurden $^{75}$. 1799 übernahm er die Leitung der „Wiener Zeitung“, die zumindest Anfang der 90er Jahre ein Organ der Gegenaufklärung war, aber bereits 1800 war er Bibliothekar an der Theresianischen Ritterakademie. 1811 fungierte er noch als Herausgeber der „Sammlung der hinterlassenen politischen Schriften“ des Prinzen Eugen von Savoyen ${ }^{76}$. 1812 starb er in Wien.

Seine Sympathie dem österreichischen Kaiserhaus gegenüber hat er schon Ende der 1780 er Jahre deutlich gemacht, wie an vielen Stellen seiner Abhandlungen zu ersehen ist. Trotz der Rigorosität der Reformpolitik Josephs II. findet dieser in weiten Teilen seinen Beifall, man kann ihn durchaus als Anhänger des Josephinismus bezeichnen. So findet er Josephs Vorgehen gegen die Kirche, sei es hinsichtlich der konfiszierten Klostergüter oder sein rigoroses Einschreiten gegen Freipräbenten und gegen Statuten der Domstifter durchaus in Ordnung. Seine leopoldinischen Annalen sind von Lobpreisungen auf Kaiser Leopold durchdrungen, aber auch die Leistungen Josephs II. werden hier noch einmal gewürdigt.

Hart ins Gericht geht er auch mit dem deutschen Reich, das für ihn keine positive Rolle in der Auseinandersetzung mit Frankreich spielte. Vor allem kritisiert er die skeptische Haltung einiger Staaten gegenüber dem österreichischen Kaiserhaus. Sartori wünscht nichts sehnlicher als die Rückkehr der alten Zustände, wie er sie in Ellwangen erlebt hatte. Deshalb schaut er mit Sorge auf alles, was eine Schwächung der inneren und äußeren Situation Österreichs bedeuten könnte, die für ihn gleichbedeutend mit der Schwächung des Reiches ist. Kaiser und Reich werden gleichgesetzt. Weniger zutreffend war hingegen seine Einschätzung der zukünftigen französischen Politik: Zwar sieht er in Frankreich den eindeutigen Sündenbock für die gegenwärtige Lage in Europa, glaubt allerdings nicht an eine weitere Expansion Frankreichs.

73 Joseph von SARTORI, Statistische Abhandlung über die Mängel in der Regierungsverfassung der geistlichen Wahlstaaten und von den Mitteln, solchen abzuhelfen, Augsburg 1787.

74 Joseph von SARTORI, Leopoldinische Annalen. Ein Beitrag zur Regierungsgeschichte Kaiser Leopolds II., 2 Bde., Augsburg 1792/93.

${ }^{75}$ Joseph von SARtori, Memoiren über die wichtigsten Staats-Materien unserer Zeit, 2 Bde., Zürich/Nürnberg 1795/97.

${ }^{76}$ Joseph von Sartori, Sammlung der hinterlassenen politischen Schriften des Prinzen Eugens von Savoyen, Stuttgart 1811. 
Das Ellwanger Stiftskapitel scheint geahnt zu haben, dass die drohende Säkularisation wohl nicht mehr zu vermeiden war. $\mathrm{Zu}$ offenkundig wurde in der zweiten Hälfte des 18. Jahrhunderts über die fehlende Zukunftsfähigkeit der geistlichen Staaten diskutiert ${ }^{77}$. Fast hilflos und verzweifelt mutet es an, dass sich das Ellwanger Stiftskapitel 1795 noch einmal alte Privilegien bestätigen ließ, die das Stift von Kaiser und Reich empfangen hatte. Genützt hat es nichts mehr. Zur selben Zeit handelten württembergische Gesandte in Paris einen Separatfrieden aus, der insgeheim die Säkularisation der geistlichen Staaten als Kompensation für Verluste links des Rheins erlaubte. Als der habsburgische Kaiser im Frieden von Campo Formio zwei Jahre später selbst die Reichskirche zur Disposition stellte, war das Ende nahe. Das Reichsoberhaupt konnte und wollte nicht länger die Existenz und Unabhängigkeit der geistlichen Staaten und damit auch Ellwangens garantieren.

\section{Kaisertum und Fürstpropstei}

Das habsburgische Reichsoberhaupt nahm als Schutzherr und Garant der Freiheiten der Reichskirche eine prominente Rolle ein. Gerade in den Sturmjahren der Reformation bildete das katholische Herrscherhaus einen nicht unerheblichen Rückhalt für die geistlichen Staaten, vor allem in Süddeutschland. So konnte ein großer Teil der Reichskirche beim alten Glauben gehalten werden ${ }^{78}$. Auch für Ellwangen wurde dieser kaiserliche Rückhalt wichtig. Geschickt verstand es das Stift, zwischen kaiserlicher Bevormundung und Durchsetzung des Eigeninteresses einen Mittelweg zu finden. Die Fürstpropstei blieb katholisch und eigenständig, auch gegen drohende württembergische Bevormundungen. Der Westfälische Friede garantierte die Reichsstandschaft, das Stift war bis zu seinem Ende fester Bestandteil der reichischen Klientel des Kaisers. Die enge Anbindung an das Haus Habsburg führte die Fürstpropstei dann seit 1689 in die große Reichskirchenpolitik. Die

77 Zur Situation der geistlichen Staaten am Ende des 18. Jahrhunderts vgl. Peter Wende, Die geistlichen Staaten und ihre Auflösung im Urteil der zeitgenössischen Publizistik, Lübeck/Hamburg 1966; Konstantin MaIeR, Die Diskussion um Kirche und Reform im Schwäbischen Reichsprälatenkollegium zur Zeit der Aufklärung, Wiesbaden 1978; Peter Hersche, Intendierte Rückständigkeit: Zur Charakteristik des geistlichen Staates im Alten Reich, in: Georg Sснміdт (Hg.), Stände und Gesellschaft im Alten Reich, Stuttgart 1989, S.133-149; Kurt Andermann, Die geistlichen Staaten am Ende des Alten Reiches, in: Historische Zeitschrift 271 (2000) S. 593-619; Ders. (Hg.), Die geistlichen Staaten am Ende des Alten Reiches. Versuch einer Bilanz, Epfendorf 2004.

78 Vgl. dazu Anton Schindling, Reichskirche und Reformation. Zu Glaubensspaltung und Konfessionalisierung in den geistlichen Fürstentümern des Reiches, in: Johannes KuNISCH (Hg.), Neue Studien zur frühneuzeitlichen Reichsgeschichte, Berlin 1987, S. 81-112; Walter Ziegler, Die Hochstifte des Reiches im konfessionellen Zeitalter 1520-1680, in: Römische Quartalschrift 87 (1992) S. 252-281; Eike WoLGaST, Hochstift und Reformation. Studien zur Geschichte der Reichskirche zwischen 1517 und 1648, Stuttgart 1995. 
Herrschaft der auswärtigen Fürstpröpste bedeutete noch einmal einen Höhepunkt für den geistlichen Barockstaat an der Jagst.

Diese Ära des barocken Reiches war durch die erfolgreiche Reichskirchenpolitik Kaiser Leopolds I. eingeleitet worden und führte dazu, dass vor und nach 1700 die meisten Fürstentümer der katholischen Reichskirche und mit ihnen auch Ellwangen fest zur Klientel der Habsburger im Reich gehörten. Mit Fürstbischöfen aus den Häusern Pfalz-Neuburg und Lothringen besetzten enge Verwandte der Kaiser Hochstifte von Breslau bis Trier. Ellwangen war in dieses Reichskirchensystem fest eingebunden. Die Fürstpröpste aus den Häusern Pfalz-Neuburg und Schönborn haben auch in Ellwangen tiefgreifende Spuren hinterlassen ${ }^{79}$.

Der Untergang der Reichskirche war ein schwerer Positionsverlust für das Haus Habsburg-Lothringen. Zur Rettung der Reichskirche hatte Wien freilich nichts unternommen, sondern stattdessen versucht, zumindest einen angemessenen Teil aus der Beutemasse für Österreich und die Sekundogeniturlinie Toskana zu ergattern. Dass auf das Ende der Reichskirche drei Jahre später das Ende des Alten Reiches erfolgte, war kein historischer Zufall, sondern unterstreicht noch einmal die enge Symbiose von Kaiser, Reich und Reichskirche. Ellwangen hatte daran einen kleinen, aber doch feinen Anteil.

79 Rudolf Reinhardt, Ellwangen als Barockstadt, in: Ellwanger Jahrbuch 33 (1989/90) S. 154-167. 